\title{
CONDICIONADORES QUÍMICOS DE SOLO E RETENÇÃO E DISTRIBUIÇÃO DE CÁDMIO, ZINCO E COBRE EM LATOSSOLOS TRATADOS COM BIOSSÓLIDO ${ }^{(1)}$
}

\author{
Maria Lucia Silveira ${ }^{(2)}$, Luís Reynaldo Ferracciú Alleoni ${ }^{(3)}$ \& Andrew Chang( ${ }^{(4)}$
}

\begin{abstract}
RESUMO
O uso agrícola é uma das alternativas eficientes para o manejo de biossólidos. Dependendo, porém, da origem (urbana e, ou, industrial) e do sistema de tratamento, biossólidos podem conter concentrações elevadas de metais pesados, que, por sua vez, podem acumular-se nos solos. O uso de condicionadores químicos pode reduzir a biodisponibilidade e a mobilidade de metais no solo e, conseqüentemente, os riscos ao meio ambiente. Neste estudo, avaliaram-se os efeitos da adição de quatro condicionadores [carbonato de cálcio $\left(\mathrm{CaCO}_{3}\right)$, sulfato de $\mathrm{Ca}\left(\mathrm{CaSO}_{4}\right)$, fosfato de potássio monobásico $\left(\mathrm{KH}_{2} \mathrm{PO}_{4}\right)$ e hidroxiapatita sintética (HA)] na retenção e distribuição de $\mathrm{Zn}, \mathrm{Cu}$ e $\mathrm{Cd}$ em Latossolos tratados com biossólido. Considerando a baixa solubilidade, a HA foi equilibrada nos valores de pH 4, 5 e 6. Utilizaram-se amostras superficiais $(0-0,2 \mathrm{~m})$ de um Latossolo Vermelho acriférrico (LVwf) e de um Latossolo Amarelo distrófico (LAd). Dois gramas de terra foram colocados para reagir em câmaras-duplas de difusão com $2 \mathrm{~g}$ de biossólidos com teores elevados de metais. Após atingir o equilíbrio (aproximadamente sete dias), a solução foi centrifugada, filtrada e acidificada, e as concentrações de $\mathrm{Zn}, \mathrm{Cu}$ e $\mathrm{Cd}$ foram determinadas. As fases sólidas (solo e biossólido) foram secas por congelamento e, a seguir, procedeu-se à extração seqüencial de $\mathrm{Zn}$, Cu e Cd. Os condicionadores foram eficientes na remoção de $\mathrm{Zn}$, $\mathrm{Cd}$ e, em menor proporção, de $\mathrm{Cu}$ da solução. Em geral, o $\mathrm{CaCO}_{3}$ foi o mais eficiente na imobilização dos metais seguido da HA (pH 6). A HA equilibrada nos valores de
\end{abstract}

\footnotetext{
(1) Recebido para publicação em maio de 2006 e aprovado em janeiro de 2008.

(2) Assistant Professor, University of Florida/IFAS Range Cattle Research and Education Center, 3401 Experiment Station, Ona, FL, USA, 33865. E-mail: mlas@ufl.br

(3) Professor Associado da Escola Superior de Agricultura "Luiz de Queiroz" - ESALQ. Caixa Postal 09, Av. Pádua Dias 11, CEP 13418-900 Piracicaba (SP). E-mail: alleoni@esalq.usp.br

(4) Função Professor, University of California, Department of Environmental Sciences, Geology Building, Riverside, CA, USA, 92521. E-mail: andrew.chang@ucr.edu
} 
pH 4 e 5 não promoveu imobilização dos metais. Após a introdução dos condicionadores, a quantidade de metais no solo ligados à fração trocável foi reduzida e os teores dos metais ligados à superfície dos óxidos/carbonatos foram aumentados, sobretudo nos tratamentos em que se utilizaram $\mathrm{CaCO}_{3}$ e $\mathrm{HA}$ (pH 6).

Termos de indexação: solos tropicais, hidroxiapatita, metais pesados, fracionamento.

\title{
SUMMARY: SOIL AMENDMENTS AND HEAVY METAL RETENTION AND DISTRIBUTION IN OXISOLS TREATED WITH BIOSOLIDS
}

\begin{abstract}
Land application represents an efficient alternative for disposal of biosolids. However, depending on the origin (urban and/or domestic) and the treatment system, biosolids may contain high amounts of heavy metals, which can gradually build up in the soil. Soil chemical amendments in contaminated areas can reduce the bioavailability and mobility of heavy metals and, consequently, minimize the risks of their adverse effects on the environment. The objective of this study was to evaluate the effects of four chemical amendments [calcium carbonate $\left(\mathrm{CaCO}_{3}\right)$, calcium sulfate $\left(\mathrm{CaSO}_{4}\right)$, monobasic potassium phosphate $\left(\mathrm{KH}_{2} \mathrm{PO}_{4}\right)$ and synthetic hydroxyapatite $\left.(\mathrm{HA})\right]$ on $\mathrm{Zn}, \mathrm{Cu}$, and $\mathrm{Cd}$ retention and distribution in Oxisols amended with biosolid. Due to the low solubility, HA was equilibrated at $\mathrm{pH} 4,5$, and 6 . Surface soil samples $(0-20 \mathrm{~cm})$ of a Rhodic Acrudox (RA) and a Typic Haplorthox (HP) were used. Two grams of each soil sample were equilibrated in a dual- diffusion chamber with $2 \mathrm{~g}$ of heavily contaminated with heavy metals. The suspension was constantly stirred for a more uniform mixing of the solutions. When the equilibrium was reached (after approximately seven days), the solution was centrifuged, filtered and acidified. Copper, $\mathrm{Zn}$, and Cd concentrations in solution were determined. The solid phases (soil and biosolid) were freeze-dried and sequential extractions of $\mathrm{Zn}, \mathrm{Cu}$ and $C d$ were performed. The chemical amendments were efficient in $\mathrm{Zn}, \mathrm{Cd}$ and, to a lesser extent, $\mathrm{Cu}$ immobilization. Calcium carbonate followed by HA (pH 6) was, in general, the most efficient treatment in reducing metal concentrations in solution. No heavy metal immobilization was obtained by $H A$ equilibrated at $p H 4$ and 5 . Chemical amendments markedly reduced the amounts of metals associated with the exchangeable fraction and increased the surface oxide/carbonate pool, especially in the treatments with $\mathrm{CaCO}_{3}$ and $H A(p H 6)$.
\end{abstract}

Index terms: tropical soils, hydroxyapatite, heavy metals, fractionation.

\section{INTRODUÇÃO}

$\mathrm{O}$ acúmulo de metais pesados em solos agrícolas tem-se tornado um problema ambiental crítico nas últimas décadas. Os metais podem ser introduzidos nos solos via deposição atmosférica, aplicação de fertilizantes, esterco animal, pesticidas e biossólidos. Embora o uso agrícola de biossólidos tenha aumentado significativamente nos últimos anos, os riscos a longo prazo dessa prática ainda são pouco conhecidos. Dependendo da origem e do sistema de tratamento do esgoto, os biossólidos podem apresentar concentrações elevadas de metais pesados. O uso agrícola de biossólidos pode reduzir as emissões de $\mathrm{CO}_{2}$ em virtude da fixação de C, com expressivos efeitos na mitigação do efeito estufa (Cerri et al., 2007). Entretanto, o uso contínuo de bissólidos com metais pesados pode levar ao acúmulo dos metais nos solos agrícolas, comprometendo a sustentabilidade do meio ambiente, além de causar sérios riscos à saúde humana (Silveira et al., 2003). Esse acúmulo de metais, embora gradual, pode ocasionar problemas na qualidade das águas de subsuperfície e na cadeia alimentar (Keller et al., 2001).

Quando aplicados ao solo, os metais nos biossólidos entram em equilíbrio com a solução e, posteriormente, com a fase sólida do solo. Dentre os principais componentes do solo envolvidos na adsorção de metais pesados, destacam-se as formas hidratadas dos óxidos de Fe e Mn, a matéria orgânica e os minerais de argila (Kabata-Pendias \& Pendias, 1987). Além dos fatores relacionados com a fração sólida do solo, a mobilidade e a disponibilidade de metais às plantas dependem da dinâmica da água no perfil do solo, da concentração iônica e do $\mathrm{pH}$ da solução do solo e da presença de ligantes orgânicos e inorgânicos (Cornu et al., 2001; Fontes \& Alleoni, 2006). 
Embora possa ser a melhor opção em alguns casos, a escavação e a remoção do material contaminado podem não ser economicamente viáveis em vastas áreas poluídas (Boisson et al., 1999). Uma alternativa economicamente adequada para recuperação de áreas contaminadas por metais pesados é a remediação in situ, que reduz os riscos de contaminação do lençol freático (Boisson et al., 1999). Nesse processo, podem ser utilizados microrganismos (biorremediação), plantas com características específicas (fitorremediação) ou compostos químicos (condicionadores de solos).

Dentre os condicionadores de solo destacam-se o calcário, o gesso agrícola, a hidroxiapatita e outras fontes solúveis de $\mathrm{P}$, materiais ricos em Fe ou Mn, e argilas, ou até mesmo biossólidos com concentrações reduzidas de metais (Boisson et al., 1999; Hamon et al., 2002; Brown et al., 2005). Calcário, fosfatos e compostos orgânicos estabilizam os metais por meio de ligações químicas, cujo resultado é a formação de compostos estáveis pouco móveis ou tóxicos, o que previne a lixiviação de metais para o lençol freático ou para outros compartimentos do ecossistema (KabataPendias \& Pendias, 1987). Os condicionadores de solos podem também causar alterações no $\mathrm{pH}$ do solo, convertendo os metais em formas não prontamente disponíveis às plantas e aos microrganismos (Hamon et al., 2002).

Estudos relacionados com o uso de condicionadores em solos contaminados por metais pesados foram, em sua maioria, desenvolvidos para solos de clima temperado. Nos solos tropicais, que, em sua maioria, exibem valores de $\mathrm{pH} \leq 5$ e reduzidos teores de matéria orgânica e de nutrientes, em especial $\mathrm{P}$, além de elevados conteúdos de óxidos de $\mathrm{Fe}$, a resposta de condicionadores de solo com relação à mobilidade e disponibilidade de metais pesados pode ser diferenciada. Nos Latossolos, por exemplo, os óxidos e hidróxidos de Fe influenciam as características adsortivas desses solos e, conseqüentemente, a retenção de metais (Silveira et al., 2002).

O objetivo deste estudo foi avaliar o efeito da utilização de condicionadores de solos na retenção e distribuição de $\mathrm{Zn}, \mathrm{Cu}$, e Cd em Latossolos tratados com biossólido.

\section{MATERIAL E MÉTODOS}

Utilizaram-se amostras da camada superficial (0-0,2 m) de dois solos do Estado de São Paulo com diferentes teores de argila e de óxidos de Fe: Latossolo Vermelho acriférrico (LVwf) e Latossolo Amarelo distrófico (LAd). Esses solos ocupam extensas áreas agrícolas e são intensamente cultivados com cana-deaçúcar. Os principais atributos químicos e físicos dos solos (Quadro 1) foram determinados de acordo com métodos descritos em Camargo et al. (1986). O biossólido foi proveniente da estação de tratamento de esgoto da cidade de Chicago, EUA, coletado em 1974 (Quadro 2). Esse biossólido foi selecionado por conter elevados teores de metais pesados, sobretudo Zn, além de teores totais de $\mathrm{Fe}$ e de $\mathrm{Al}$ semelhantes aos encontrados em biossólidos produzidos no Brasil (Fe total $=31 \mathrm{~g} \mathrm{~kg}^{-1} ; \mathrm{Al}$ total $\left.=26 \mathrm{~g} \mathrm{~kg}^{-1}\right)$.

Após a coleta, as amostras de terra e de biossólido foram secas ao ar, moídas em mortar de ágata e passadas por peneira de 100 mesh de malha. Aproximadamente 0,25 g de terra e $0,1 \mathrm{~g}$ de biossólido foram pesados e procedeu-se à digestão completa das amostras em microondas, utilizando o método EPA $3052\left(\mathrm{HNO}_{3}, \mathrm{HF}\right.$ e água deionizada) (USEPA, 1996). No caso da amostra de biossólidos, antes da adição do ácido nítrico, adicionou-se $1 \mathrm{~mL}$ de $\mathrm{H}_{2} \mathrm{O}_{2}$ $30 \%$ para solubilizar o material orgânico. A concentração de metais nas amostras de terra e de biossólidos encontra-se no quadro 2.

Quadro 2. Concentração total ${ }^{(1)}$ de $\mathrm{Zn}, \mathrm{Cu}$ e Cd nas amostras de solo e de biossólido

\begin{tabular}{crcc}
\hline Solo/biossólido & $\mathbf{Z n}$ & $\mathbf{C u}$ & $\mathbf{C d}$ \\
\hline & & $\mathrm{mg} \mathrm{kg}^{-1}$ & \\
\cline { 2 - 4 } & & & \\
LVwf & 184 & 257 & 0,22 \\
LAd & 13 & 13 & 0,15 \\
Biossólido & 4.319 & 1.263 & 152 \\
\hline
\end{tabular}

(1) Método EPA 3052 (USEPA, 1996). LVwf: Latossolo Vermelho acriférrico; LAd: Latossolo Amarelo distrófico.

\section{Quadro 1. Atributos químicos e físicos dos solos}

\begin{tabular}{|c|c|c|c|c|c|c|c|c|c|c|c|c|c|c|}
\hline Solo & pH $\mathrm{H}_{2} \mathrm{O}$ & C & $\mathrm{Ca}^{2+}$ & $\mathbf{M g}^{2+}$ & $\mathbf{K}^{+}$ & $\mathrm{Na}^{+}$ & $\mathrm{Al}^{3+}$ & CTC & Areia & Silte & Argila & $\mathrm{Fe}_{\mathrm{H}_{2} \mathrm{SO}_{4}}$ & $\mathrm{Fe}_{\mathrm{d}}$ & $F e_{o}$ \\
\hline & & $\mathrm{g} \mathrm{kg}^{-1}$ & \multicolumn{6}{|c|}{$-\mathrm{mmol}_{\mathrm{c}} \mathrm{dm}^{-3}$} & \multicolumn{6}{|c|}{$-\mathrm{g} \mathrm{kg}^{-1}$} \\
\hline LVwf & 5,0 & 20 & 40 & 17 & 5,8 & 0,1 & 0 & 62,9 & 170 & 230 & 600 & 220 & 124 & 0,7 \\
\hline LAd & 4,0 & 8,3 & 0,4 & 0,1 & 0,02 & 0 & 1,43 & 2,0 & 830 & 50 & 120 & 9 & 3,7 & 0,06 \\
\hline
\end{tabular}

LVwf: Latossolo Vermelho acriférrico; LAd: Latossolo Amarelo distrófico; $\mathrm{Fe}_{\mathrm{H} 2 \mathrm{SO} 4}$ : teor de Fe obtido após ataque sulfúrico; Fe

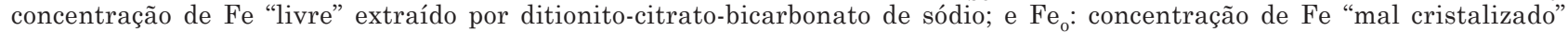
extraído por oxalato de sódio a pH 3. 
Amostras de solos e biossólido foram equilibradas em câmaras-duplas de difusão (CDD) adaptadas de DePinto (1982). Esse aparato consiste de duas câmaras de policarbonato separadas por uma membrana seletiva de difusão (Figura 1). Em uma das unidades da câmara é adicionada a amostra de terra e, na outra, o biossólido. Ambas as unidades são preenchidas com solução eletrolítica, com concentração próxima à força iônica da solução do solo, e um sistema de agitação possibilita que as fases permaneçam em suspensão. Após montadas as duas unidades das CDD, o conjunto apresenta aproximadamente $23 \mathrm{~cm}$ de comprimento e volume de $300 \mathrm{~cm}^{3}$. A membrana que separa as duas unidades apresenta poros de diâmetro de $0,45 \mu \mathrm{m}$ e permite a passagem de metais na forma solúvel entre as duas unidades das câmaras, separando-os da fração coloidal (Ross, 1994). A utilização das CDD permite avaliar a dinâmica dos metais pesados no sistema solo-biossólido, além de possibilitar a caracterização das fases sólidas e da solução após as reações de equilíbrio (Candelaria \& Chang, 1997; Silveira et al., 2007a).

Dois gramas de solo ou biossólidos (equivalente ao material seco) foram colocados em cada um dos lados das CDD, como descrito em Silveira et al. (2007a). As CDDs foram preenchidas com $500 \mathrm{~mL}$ de solução salina composta de $\mathrm{Ca}\left(\mathrm{NO}_{3}\right)_{2} 0,005 \mathrm{~mol} \mathrm{~L}^{-1}+\mathrm{CaSO}_{4}$ $0,003 \mathrm{~mol} \mathrm{~L}^{-1}$. Essa solução foi preparada com base nas características químicas da solução dos solos estudados, extraída pelo método da pasta de saturação (Rhoades, 1996). A força iônica foi calculada a partir da condutividade elétrica da solução de acordo com a equação 1 (Griffin \& Jurinak, 1973).

$$
\mathrm{I}_{\mathrm{s}}=\mathrm{CE} \times 0,013
$$

em que $\mathrm{I}_{\mathrm{S}}=$ força iônica; $\mathrm{CE}$ = condutividade elétrica da solução.

Os seguintes condicionadores de solo foram adicionados às câmaras: $\mathrm{CaCO}_{3}, \mathrm{CaSO}_{4} ; \mathrm{KH}_{2} \mathrm{PO}_{4} \mathrm{e}$ hidroxiapatita sintética (HA). As doses foram equivalentes a $1 \%$ da massa de solo para $\mathrm{CaCO}_{3}$, $\mathrm{CaSO}_{4}$, e HA. O KH${ }_{2} \mathrm{PO}_{4}$ foi escolhido por ser uma fonte solúvel de $\mathrm{P}$, e sua quantidade adicionada foi

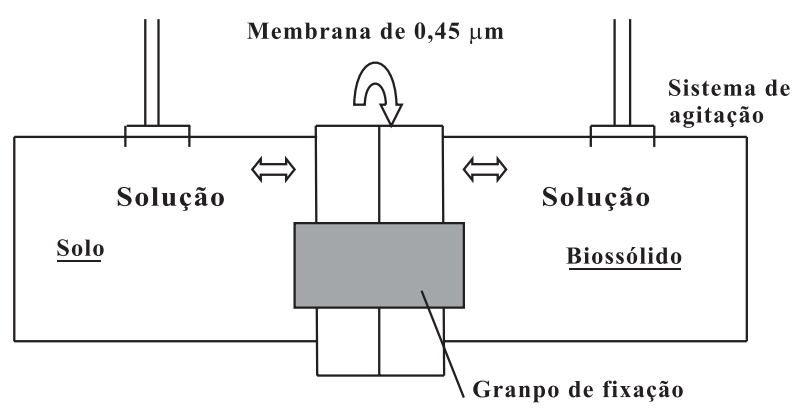

Figura 1. Representação esquemática das câmaras duplas de difusão (CDD). baseada na percentagem de $\mathrm{P}$ nos tratamentos com HA. Considerando sua baixa solubilidade [produto de solubilidade $\left(\mathrm{K}_{\mathrm{ps}}=2,34.10^{-59}\right)$ ], a HA foi equilibrada em três valores de $\mathrm{pH}(4 ; 5$; e 6$)$, para verificar o efeito da sua dissolução na retenção e distribuição de metais nos solos. Os valores de $\mathrm{pH} 4$ e 6 foram ajustados ou com $\mathrm{HCl} 0,1 \mathrm{~mol} \mathrm{~L}^{-1}$ ou com $\mathrm{NaOH} 0,1 \mathrm{~mol} \mathrm{~L}^{-1}$, respectivamente. Nos demais tratamentos $\left(\mathrm{CaCO}_{3}\right.$, $\mathrm{CaSO}_{4}$, e $\mathrm{KH}_{2} \mathrm{PO}_{4}$ ), o $\mathrm{pH}$ da solução não foi ajustado. $\mathrm{O} \mathrm{pH}$ da solução dos tratamentos que receberam $\mathrm{CaSO}_{4}, \mathrm{KH}_{2} \mathrm{PO}_{4}$ e das amostras controle mantiveramse ao redor de 5. Para o tratamento que recebeu $\mathrm{CaCO}_{3}$, o pH da solução variou de 6,5 a 7,0. Um conjunto "testemunha" foi avaliado paralelamente, composto apenas pelo solo e pelo biossólido, equilibrados sem adição dos condicionadores. Como as quantidades de HA utilizadas em áreas contaminadas não são bem definidas, foram testadas previamente doses entre 1 e $2 \%$ (base massa) de HA. Em estudos preliminares, observou-se que doses de HA superiores a $2 \%$ não promoveram diminuição na disponibilidade de metais em solução. Portanto, doses inferiores a $2 \%$ foram selecionadas.

A suspensão em ambos os lados das câmaras (solo + condicionadores e biossólidos) foi agitada constantemente e o $\mathrm{pH}$ e a $\mathrm{CE}$ foram medidos diariamente até que o equilíbrio fosse atingido. O equilíbrio foi definido quando ambos os lados das câmaras apresentaram os mesmos valores de $\mathrm{pH}$ e CE, o que foi obtido aproximadamente em sete dias. Os valores iniciais de $\mathrm{pH}$ e da força iônica da solução não foram alterados após os sete dias de equilíbrio nas câmaras (resultados não mostrados). Após atingir o equilíbrio, as câmaras foram desmontadas, as amostras de terra e de biossólidos foram secas por meio de congelamento e, em seguida, separaram-se duas subamostras: uma para realizar a digestão completa de acordo com o método EPA 3052 (USEPA, 1996) e outra para proceder à extração seqüencial, utilizando-se o método proposto por Silveira et al. (2007b). A extração seqüencial caraterizou os metais associados às seguintes frações: (a) trocável (Troc.); (b) superfície de óxidos (Superf. Ox.); (c) matéria orgânica (MO); (d) óxidos de $\mathrm{Mn}(\mathrm{Ox} . \mathrm{Mn})$; (e) óxidos de $\mathrm{Fe}$ mal-cristalizados (Ox. Fe); (f) óxidos de Fe cristalinos (Fe Crist.); e (g) residual (Res.).

A solução das câmaras foi centrifugada a $3.500 \mathrm{rpm}$ por $10 \mathrm{~min}$, filtrada $(0,45 \mu \mathrm{m}) \mathrm{e}$, posteriormente, acidificada com $\mathrm{HNO}_{3}$ concentrado. Adicionou-se $0,5 \mathrm{~mL}$ de $\mathrm{HNO}_{3}$ concentrado em $500 \mathrm{~mL}$ de solução. As concentrações de $\mathrm{Zn}$ e $\mathrm{Cu}$ foram determinadas num espectrofotômetro de absorção atômica (chama de aracetileno). Os teores de $\mathrm{Cd}$ foram determinados em forno de grafite. Na determinação de $\mathrm{Cd}$, foram adicionados os seguintes modificadores de matriz: $\mathrm{Mg}\left(\mathrm{NO}_{3}\right)_{2} 0,06 \%$ e $\mathrm{NH}_{4} \mathrm{H}_{2} \mathrm{PO}_{4} 1 \%$ para favorecer o processo de pirólise da amostra. Amostras certificadas de água (NIST 1640 - Trace Elements in Natural Water), solo (NIST 2709 - San Joaquin Soil) e biossólidos (NIST 2781- Domestic Sludge) foram utilizadas para assegurar a qualidade das análises. 
As amostras de solo, biossólidos e solução foram analisadas em duplicata. Calculou-se o balanço de massa de $\mathrm{Zn}, \mathrm{Cu}$ e $\mathrm{Cd}$ nas três fases após o equilíbrio nas câmaras (solução, solo e biossólido). O grau de recuperação (100 $\pm 20 \%$ ) foi utilizado como critério de controle de qualidade das análises. Foi feita a comparação de médias de acordo com o método de Tukey $(\mathrm{p}<0,05)$ para avaliar o efeito dos condicionadores na distribuição de $\mathrm{Zn}, \mathrm{Cu}$, e $\mathrm{Cd}$ na fase sólida (solo e biossólido) e em solução.

\section{RESULTADOS E DISCUSSÃO}

\section{Distribuição dos metais na fase sólida e líquida após o equilíbrio nas câmaras}

A adição dos condicionadores alterou a distribuiçãa de $\mathrm{Zn}$ e $\mathrm{Cd}$ na fase sólida e em solução (Figura 2). Esse efeito foi menos acentuado para o $\mathrm{Cu}$, que praticamente não teve sua solubilidade afetada pelo uso de condicionadores. $\mathrm{O} \mathrm{Cu}$ parece estar retido mais fortemente na matriz do biossólido em relação aos demais metais, graças, possivelmente, à sua forte interação com os óxidos de Fe e com a matéria orgânica presente nesse resíduo (Smith, 1994a,b).

As quantidades de Zn e Cd em solução diminuíram após a adição dos condicionadores, exceto quando HA foi equilibrada no pH 4 (Figura 2). No LAd, o equivalente a $4.017 \mu \mathrm{g}$ de $\mathrm{Zn}$ (48 \% do Zn total) estava presente em solução na câmara equilibrada sem o uso de condicionadores. Porém, com a introdução de $\mathrm{CaSO}_{4}, \mathrm{KH}_{2} \mathrm{PO}_{4}$ e HA (pH 5), a massa de $\mathrm{Zn}$ em solução foi reduzida para 1.512; 2.472 e $2.138 \mu \mathrm{g}$, respectivamente. Resultados semelhantes foram obtidos para Cd: em geral, houve redução de 51 \% na quantidade do metal em solução após a adição de condicionadores. Ma et al. (1994) observaram que a HA apresentou capacidade de reter os metais na seguinte ordem: $\mathrm{Al}>\mathrm{Zn}>\mathrm{Fe}(\mathrm{II})>\mathrm{Cd}>\mathrm{Cu}>\mathrm{Ni}$. Concordando com essa seqüência de afinidade proposta por Ma et al. (1994), as quantidades de Zn e o Cd em solução foram alteradas mais acentuadamente do que o $\mathrm{Cu}$ quando se adicionou HA. A redução nas quantidades dos metais em solução foi devida à incorporação ou coprecipitação desses elementos nos sítios de adsorção de $\mathrm{Ca}$ da $\mathrm{HA}$ ou nos radicais hidroxílicos da superfície da HA.

A adição de $\mathrm{CaCO}_{3}$ e o tratamento $\mathrm{HA}(\mathrm{pH}$ 6) promoveram menor solubilização dos metais do biossólido e, conseqüentemente, em solução (Figura 2). Em termos gerais, o $\mathrm{CaCO}_{3}$ foi o condicionador mais eficiente na redução da disponibilização de metais do biossólido para solução, sobretudo $\mathrm{Zn}$ e $\mathrm{Cd}$, (Figura 2a,c). Para ambos os solos, a percentagem de $\mathrm{Zn}$, Cu e Cd em solução variou de 0 a $3 \%$ do total nas amostras tratadas com $\mathrm{CaCO}_{3}$ e de 0 a $4 \%$ no tratamento com HA (pH 6). Fang \& Wong (1999) observaram que a adição de $\mathrm{CaCO}_{3}$ reduziu as quantidades de metais solúveis $(\mathrm{Cd}, \mathrm{Cr}$ e $\mathrm{Pb})$ e trocáveis (Cu, Mn, Ni e Zn) presentes em biossólidos. Porém, Fang \& Wong (1999) indicaram o uso de doses de $\mathrm{CaCO}_{3}$ inferiores a $1 \%$ (base massa), pois doses mais elevadas podem promover perdas de $\mathrm{N}$ do biossólido via volatilização. A redução nos teores dos metais em solução em resposta ao uso de $\mathrm{CaCO}_{3}$ deve-se ao aumento no número de sítios na fase sólida capazes de retê-los ou elevar a energia da ligação (Hamon et al., 2002). Os teores de $\mathrm{Cu}$ e Zn trocáveis atribuídos ao tratamento com $\mathrm{CaCO}_{3}$ obtidos por Fang \& Wong (1999) foram iguais a 45 e $70 \%$, respectivamente, quando comparados aos da amostra-controle, enquanto para o $\mathrm{Cd}$ não foi detectada a presença desse metal na fração trocável após o tratamento.

Hamon et al. (2002) observaram que o $\mathrm{KH}_{2} \mathrm{PO}_{4}$ foi mais eficiente na diminuição da concentração de $\mathrm{Cd}$ e $\mathrm{Zn}$ em solução do que o $\mathrm{CaCO}_{3}$. Esses resultados diferem dos obtidos no presente trabalho. Possivelmente, a maior quantidade de $\mathrm{KH}_{2} \mathrm{PO}_{4}$ utilizada no trabalho de Hamon et al. (2002) $\left(20 \mathrm{~g} \mathrm{~kg}^{-1}\right.$ de $\mathrm{P}$ no solo) e a correção do $\mathrm{pH}$ com $\mathrm{CaO}$ para 7,2 resultaram em maior imobilização de metais. Hamon et al. (2002), porém, ressaltaram que as quantidades de metais em solos tratados com $\mathrm{KH}_{2} \mathrm{PO}_{4}$ aumentaram exponencialmente com a redução do $\mathrm{pH}$.

Ao contrário do observado por Ma et al. (1993), a redução do $\mathrm{pH}$ nos tratamentos que receberam HA não ocasionou menor solubilização dos metais em solução. A diminuição do $\mathrm{pH}$ para 4 no tratamento com HA promoveu dissolução mais acentuada de Zn e $\mathrm{Cd}$ e, em menor proporção, de $\mathrm{Cu}$ do biossólido. Como conseqüência, houve maior concentração desses metais em solução nesse tratamento em relação aos demais tratamentos. Esses resultados descartam a hipótese de que, em menores valores de $\mathrm{pH}$, a dissolução da HA é favorecida (Kanabo \& Gilkes, 1987), resultando em maior concentração de $\mathrm{PO}_{4} \mathrm{e}$, conseqüentemente, em maior complexação dos metais em solução.

$\mathrm{O}$ efeito direto do $\mathrm{pH}$ da solução na solubilização de metais mostrou-se mais importante do que a percentagem de dissolução da HA. Na especiação obtida com uso do programa Visual Minteq (Gustafsson, 2002), os índices de saturação indicaram que a HA estava totalmente dissolvida, mesmo em valores de $\mathrm{pH}$ mais elevados (índices de saturação da $\mathrm{HA}=-45,8,-38,9 \mathrm{e}-32,1$ para os valores de $\mathrm{pH} 4,5 \mathrm{e}$ 6 , respectivamente). Esse efeito poderia ter sido mais bem evidenciado se fossem utilizadas doses mais elevadas de HA e, ou, de metais em solução. Ma et al. (1993) observaram que a reação de 0,2 g de HA com uma solução que continha $500 \mathrm{mg} \mathrm{L}^{-1}$ de $\mathrm{Pb}$ foi suficiente para formar hidroxipiromorfita, tendo a redução do pH da solução favorecido a formação desse mineral. Todavia, Boisson et al. (1999) relataram que o aumento no $\mathrm{pH}$ da solução favoreceu os mecanismos de troca iônica e de complexação de superfície entre os metais e a HA. Além disso, Boisson et al. (1999) 
observaram que, na dose de $1 \%$, a $\mathrm{HA}(\mathrm{pH} 5,6)$ foi capaz de reduzir significativamente os teores trocáveis de $\mathrm{Cd}, \mathrm{Pb}, \mathrm{Cu}$ e Zn no solo. Xu \& Schwartz (1994) encontraram aumento na adsorção de $\mathrm{Cd}$ e $\mathrm{Zn}$ no tratamento com HA na faixa de $\mathrm{pH}$ entre 5,4 e 6,9. Portanto, o efeito do $\mathrm{pH}$ parece depender das quantidades de HA e de metais em solução. O aumento na acidez pode favorecer a dissolução da HA, enquanto a elevação do $\mathrm{pH}$ favorece os mecanismos de troca nesse material. Uma vez que a dose de HA foi totalmente dissolvida, mesmo nos $\mathrm{pHs}$ mais elevados, verifica-se que o segundo mecanismo foi mais importante nesse experimento.

\section{Efeito dos solos no equilíbrio de metais no sistema biossólido-solução}

De forma geral, as quantidades de metais retidas no biossólido foram superiores na amostra equilibrada com o LVwf quando comparada ao LAd, sobretudo para

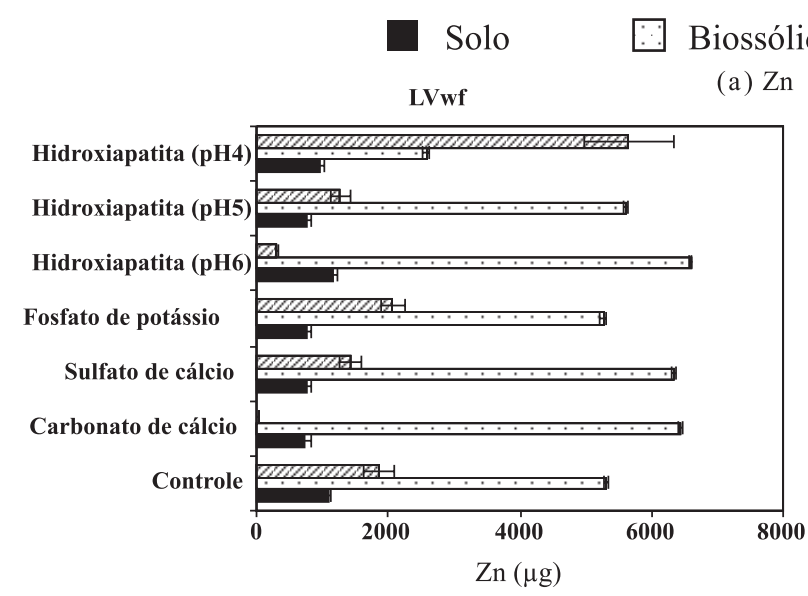

a) $\mathrm{Zn}$

Solução

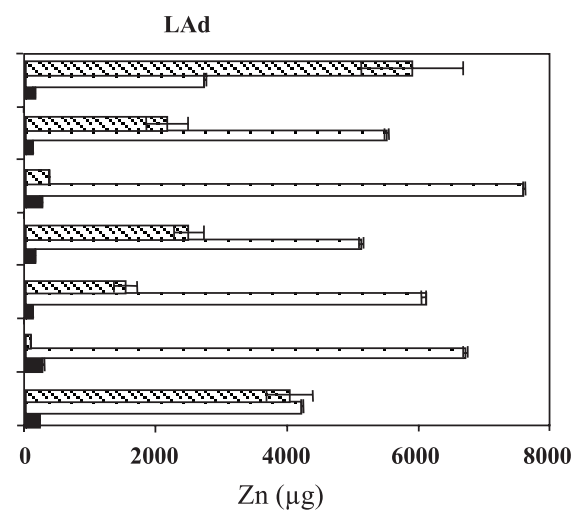

LVwf

(b) $\mathrm{Cu}$
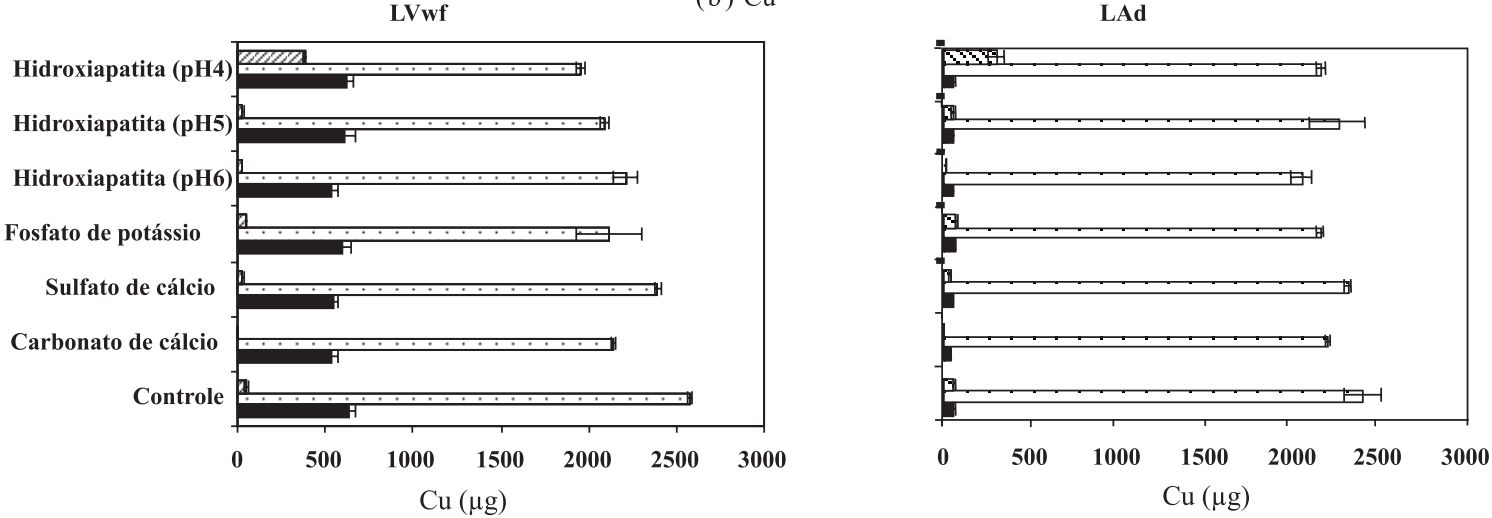

LVwf

(c) $\mathrm{Cd}$
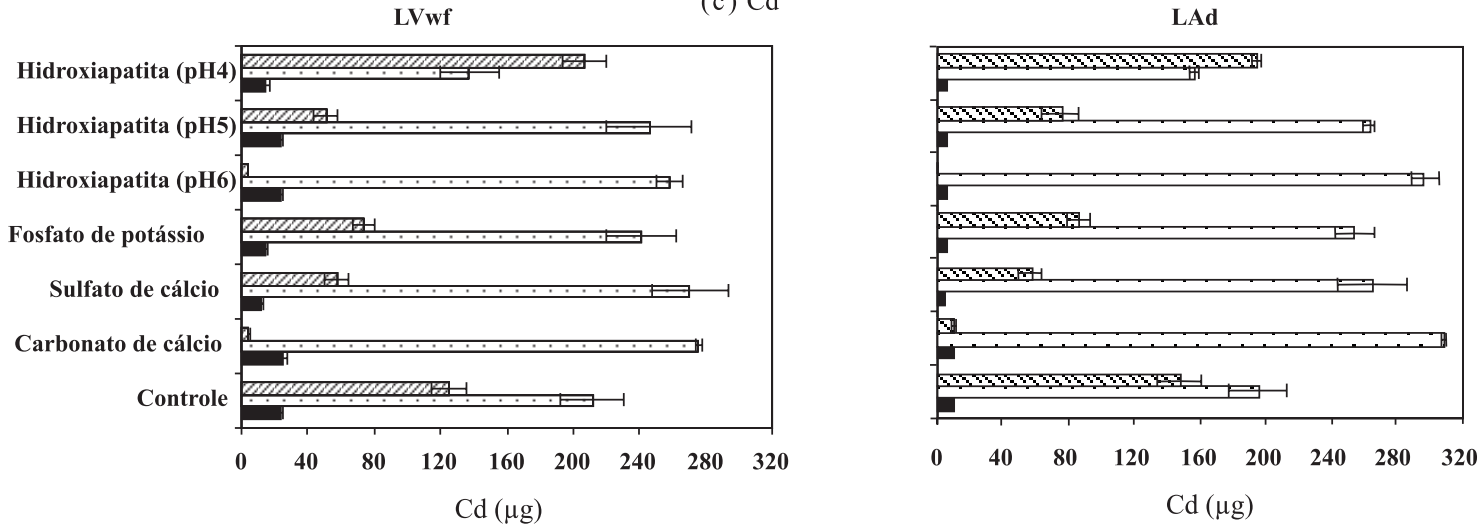

Figura 2. Distribuição de zinco, cobre e cádmio na fase sólida ( $2 \mathrm{~g}$ de terra e de biossólido) e em solução $(500 \mathrm{~mL})$ após o equilíbrio nas câmaras duplas de difusão. Controle: amostras sem adição de condicionadores; LVwf: Latossolo Vermelho acriférrico; LAd: Latossolo Amarelo distrófico. Barras representam desvio-padrão. 
o $\mathrm{Cu}$ (Figura 3). Isso pode ser atribuído à maior capacidade do LVwf em adsorver metais em decorrência dos teores elevados de argila e de óxidos de Fe (Quadro 1). A importância dos óxidos de Fe na retenção de metais nesses solos foi também observada por Silveira et al. (2002). A menor concentração de metais em solução no LVwf (Figura 2) confirma a hipótese de que os atributos do solo podem influenciar a reação de dissolução do biossólido.

Entre 65 e $80 \%$ do $\mathrm{Zn}$ total permaneceu no biossólido nos tratamentos equilibrados com $\mathrm{CaSO}_{4}$, $\mathrm{KH}_{2} \mathrm{PO}_{4}$ e HA (pH 5) em ambos os solos (Figura 3a). A adição dos condicionadores reduziu a disponibilização de metais no biossólido equilibrado com o LAd, em que $50 \%$ do Zn total ficou retido no biossólido. Em contrapartida, quando se utilizaram $\mathrm{CaSO}_{4}, \mathrm{KH}_{2} \mathrm{PO}_{4}$ e HA (pH 5), os valores foram aumentados para cerca de $70 \%$ do Zn total. Em geral, as quantidades de Zn solubilizadas no biossólido equilibrado com o LVwf foram superiores àquelas equilibradas com o LAd. Por exemplo, nos tratamentos com HA ( $\mathrm{pHs} 6$ e 4), as quantidades do metal retidas no biossólido foram de 82 e $28 \%$, no LVwf, e de 93 e $31 \%$, no LAd, respectivamente. Possivelmente, em virtude da maior capacidade de adsorção do LVwf, o equilíbrio da reação foi deslocado, favorecendo a maior dissolução do biossólido. No tratamento com $\mathrm{CaCO}_{3}$, a maior parte do Zn ficou no biossólido (96 \% do total no LAd e $89 \%$ no LVwf).

A adição dos condicionadores não alterou a dissolução de $\mathrm{Cu}$ do biossólido (Figura 3b). Na amostra equilibrada com o LVwf, cerca de $80 \%$ do metal ficou retido tanto na amostra-controle quanto nas amostras tratadas com os condicionadores, com exceção do tratamento HA ( $\mathrm{pH} 4)$, no qual ficou retido $66 \%$ do $\mathrm{Cu}$ total no biossólido. Na amostra que reagiu com o LAd, quase todo $\mathrm{Cu}$ ficou retido no biossólido, independentemente do tratamento testado, mesmo no HA (pH 4), em que, aproximadamente, $86 \%$ permaneceu no biossólido. $\mathrm{O} \mathrm{Cu}$ foi o metal menos influenciado pela adição dos condicionadores e, ou, pela variação do pH da solução, o que se deveu, possivelmente, à elevada afinidade da superfície dos colóides em reter esse metal e ao efeito menos acentuado das mudanças no $\mathrm{pH}$ na disponibilização de $\mathrm{Cu}$ em solos tratados com biossólidos (Smith, 1994a).

A quantidade de $\mathrm{Cu}$ disponibilizada pelo biossólido equilibrado com o LVwf foi superior em todos os tratamentos, quando comparada à do LAd. Embora ambos os Latossolos apresentem baixos teores de $\mathrm{C}$ orgânico, possivelmente o maior teor no LVwf (Quadro 1) pode ter resultado em maior quantidade de ligantes orgânicos em solução, o que favoreceu a dissolução do Cu do biossólido. Salam \& Helmke (1998) observaram que a quantidade de $\mathrm{Cu}$ em solução era mais afetada, em relação ao $\mathrm{Cd}$, pela quantidade de ligantes orgânicos em solução.
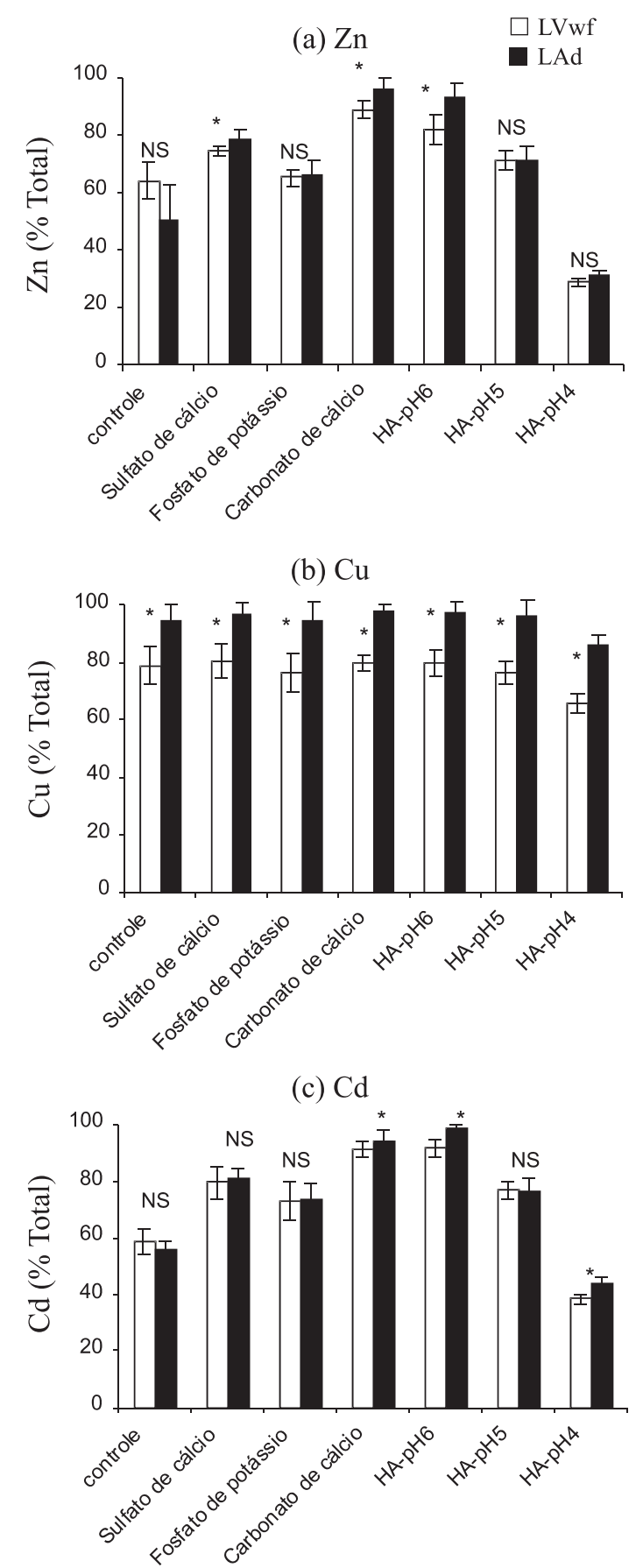

Figura 3. Percentagem dos metais retidos nas amostras de biossólido após o equilíbrio com o Latossolo Vermelho acriférrico (LVwf ) e o Latossolo Amarelo distrófico (LAd). Análises estatísticas referem-se à comparação entre solos, para um mesmo condicionador. * F significativo a $5 \%$. NS: diferença não-significativa.

Com exceção do HA (pH 4), todos os tratamentos ocasionaram menor disponibilização de $\mathrm{Cd}$ do biossólido (Figura 3c). Cerca de $60 \%$ do Cd estava presente nas amostras de biossólido antes da adição dos condicionadores. Porém, após adição de $\mathrm{CaSO}_{4}$, 
$\mathrm{KH}_{2} \mathrm{PO}_{4}$ e $\mathrm{HA}(\mathrm{pH}$ 5), esse valor variou de 73 a $79 \%$ no LVwf e de 74 a $81 \%$ no LAd. Os tratamentos HA (pH 6) e $\mathrm{CaCO}_{3}$ elevaram a quantidade de $\mathrm{Cd}$ retida no biossólido para até $98 \%$ do total, graças, possivelmente, à elevação do $\mathrm{pH}$ da solução.

\section{Distribuição de zinco na fase sólida}

Nas amostras-controle, o $\mathrm{Zn}$ foi encontrado principalmente na fração residual (Res) no LVwf (30 \% do total) e na forma trocável (Troc) no LAd (78 \% do $\mathrm{Zn}$ total) (Figura 4). A maior capacidade do LVwf em reter o Zn em formas pouco móveis se deveu, sobretudo, aos maiores teores de argila e óxidos de Fe cristalino (Quadro 1).

A adição dos condicionadores diminuiu as quantidades de $\mathrm{Zn}$ retidas na fração Troc. em ambos os solos e elevou os teores do metal obtidos na fração associada à superfície dos óxidos (Figura 4a,b). Esse efeito foi mais pronunciado quando se utilizou $\mathrm{CaCO}_{3}$. Após a adição desse condicionador, o LVwf teve redução de 22 para $0 \%$ do $\mathrm{Zn}$ associado à fração Troc., enquanto, no LAd, a redução foi de 78 para $3 \%$. Paralelamente, houve aumento de 10 a $27 \%$ e de 13 a
$68 \%$ do Zn total retido na fração Superf.Ox. no LVwf e no LAd, respectivamente. A elevação do $\mathrm{pH}$ atribuída ao uso de $\mathrm{CaCO}_{3}$ resulta na transferência dos metais para formas moderadamente lábeis, as quais apenas estão presentes em valores de $\mathrm{pH}$ elevados, tais como na fração carbonato (Hamon et al., 2002). Entretanto, graças às formas de $\mathrm{Zn}$ predominantes no tratamento com $\mathrm{CaCO}_{3}$, a possível reacidificação posterior poderá resultar na disponibilização do Zn para solução.

Em seguida ao $\mathrm{CaCO}_{3}$, o tratamento $\mathrm{HA}(\mathrm{pH}$ 6) foi o que ocasionou maior redução na percentagem de $\mathrm{Zn}$ retido na forma trocável. Após a adição da HA-pH 6, cerca de 12 e $40 \%$ do Zn total estavam retidos na fração Troc. no LVwf e no LAd, respectivamente. As demais frações não sofreram alterações após adição dos condicionadores. Boisson et al. (1999) observaram que o uso da HA foi capaz de reduzir os teores trocáveis dos metais em solos contaminados para valores inferiores aos encontrados em amostras-controle (não contaminadas). Esse efeito foi mais acentuado para $\mathrm{Zn}$ e $\mathrm{Cd}$ do que para o $\mathrm{Cu}$. Isso, provavemente, se deveu ao fato de o raio iônico do $\mathrm{Cd}(0,097 \mathrm{~nm})$ ser mais próximo ao do $\mathrm{Ca}(0,094 \mathrm{~nm})$, o que pode ter favorecido a substituição isomórfica entre esses cátions (a) LVwf

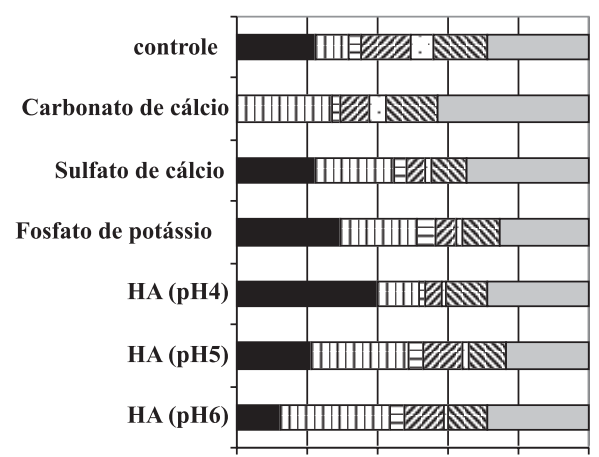

(b) LAd

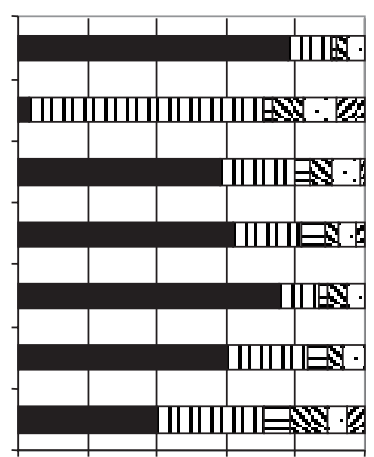

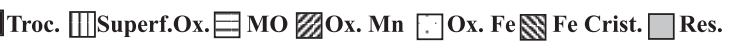

(c) Biossólido 1

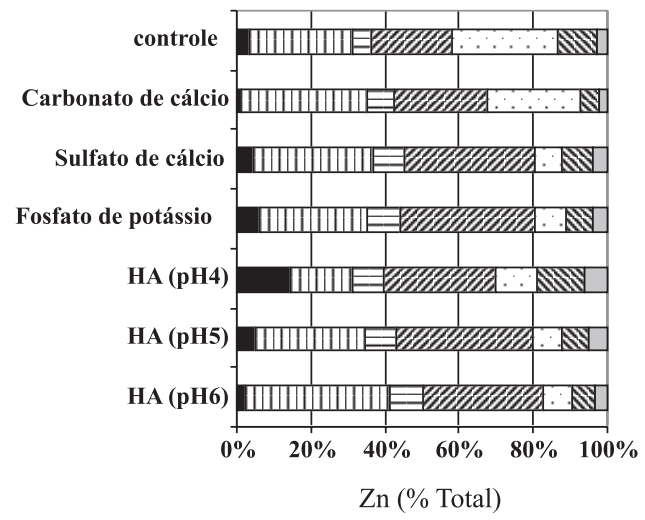

(d) Biossólido 2

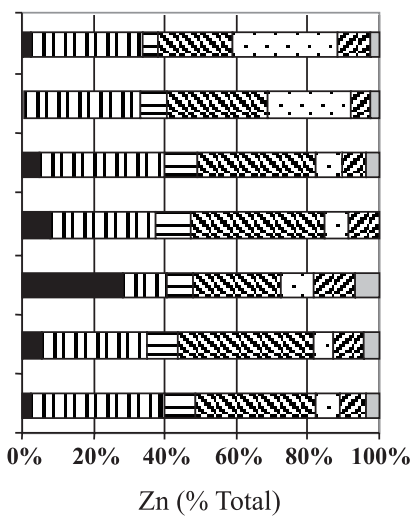

Figura 4 Distribuição das formas de zinco nas amostras de solo e biossólido após os equilíbrios nas câmaras duplas de difusão. LVwf: Latossolo Vermelho acriférrico; LAd: Latossolo Amarelo distrófico; Biossólido 1: biossólido equilibrado com LVwf; Biossólido 2: biossólido equilibrado com LAd; Troc.: trocável; Superf. Ox.: superfície de óxidos; MO: matéria orgânica; Ox. Mn: óxidos de Mn; Ox. Fe: óxidos de Fe mal cristalizados; Fe Crist.: óxidos de Fe cristalinos; Res.: residual. 
na hidroxiapatita em relação ao $\mathrm{Zn}$ (cujo raio iônico é próximo de $0,074 \mathrm{~nm})$ e ao $\mathrm{Cu}(0,069 \mathrm{~nm})$ (Boisson et al., 1999). O Ca serve como referência porque é o cátion na hidroxiapatita, podendo ser substituído por metais de tamanho semelhante. Corroborando esses resultados, Xu \& Schwartz (1994) observaram que maiores quantidades de $\mathrm{Cd}$ eram retidas na HA em relação ao $\mathrm{Zn}$. Porém, embora o $\mathrm{Cd}$ fosse retido em maior proporção, a quantidade de $\mathrm{Zn}$ dessorvida foi menor, sugerindo que esse último metal foi retido mais fortemente na HA.

A adição de $\mathrm{CaSO}_{4}, \mathrm{KH}_{2} \mathrm{PO}_{4}$ e $\mathrm{HA}$ resultou em menor quantidade de $\mathrm{Zn}$ associado à fraçãoTroc. apenas no LAd, em que a redução foi ao redor de $20 \%$, seguida de aumento médio de $70 \%$ na fração Superf. Ox. (Figura 4). Já para o LVwf, não foi observada diferença na distribuição de $\mathrm{Zn}$ após adição de $\mathrm{CaSO}_{4}$, $\mathrm{KH}_{2} \mathrm{PO}_{4} \mathrm{e} \mathrm{HA}(\mathrm{pH}$ 5), possivelmente pela participação da fração residual e ligada aos óxidos de $\mathrm{Fe}$ na retenção desse metal. $\mathrm{O}$ tratamento com $\mathrm{KH}_{2} \mathrm{PO}_{4}$ no $\mathrm{LVwf}$ promoveu ligeiro aumento na quantidade de $\mathrm{Zn}$-Troc. de 22 para $30 \%$ (amostra-controle e tratada, respectivamente). Resultados semelhantes foram observados por Shuman (1988), o qual concluiu que a adição de $\mathrm{KH}_{2} \mathrm{PO}_{4}$, nas doses de $30-60 \mathrm{mg} \mathrm{kg}^{-1}$, era capaz de aumentar a quantidade do metal trocável em solos com $\mathrm{pH}$ próximos ao encontrado no LVwf.

A distribuição de Zn nos biossólidos equlibrados com o LVwf ou com o LAd (Figura 4c,d) foi semelhante. A maior parte do metal ficou retida nas frações Superf. Ox. e Ox. Mn. O efeito dos condicionadores foi mais evidente no tratamento com $\mathrm{CaCO}_{3}$, o qual resultou na diminuição da fração Zn-Troc. (3 para 1 \% do Zn total) (Figura 4c,d). Resultados semelhantes foram obtidos por Fang \& Wong (1999) e Wong \& Fang (2000). Os autores encontraram redução nos teores de Zn-trocável em amostras de biossólido tratadas com $\mathrm{CaCO}_{3}$. Os demais tratamentos não alteraram a distribuição de Zn no biossólido.

\section{Distribuição de cobre na fase sólida}

$\mathrm{O} \mathrm{Cu}$ foi encontrado principalmente na fração residual no LVwf e adsorvido na superfície dos óxidos no LAd (Figura 5). Na amostra-controle do LVwf, cerca de $53 \%$ do Cu total estava retido na fração Res. e $16 \%$ na Ox. Mn. Já no LAd, $50 \%$ do Cu total foi (a) $\mathbf{L V w f}$



(c) Biossólido 1



(b) LAd

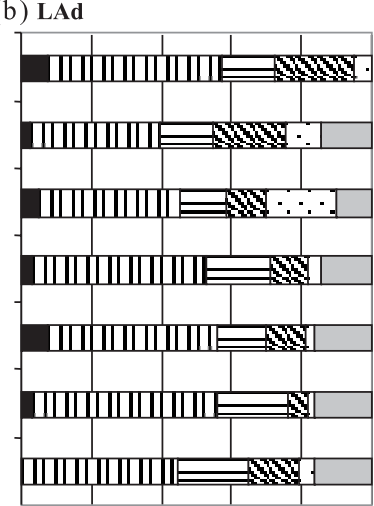

Ox. Fe Fe Crist. $\square$ Res.

(d) Biossólido 2

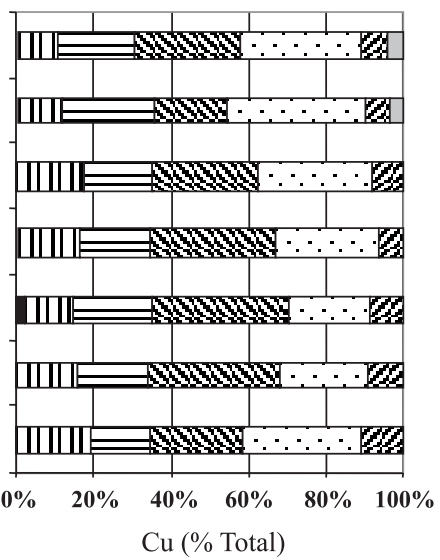

Figura 5. Distribuição das formas de cobre nas amostras de solo e biossólido após os equilíbrios nas câmaras duplas de difusão. LVwf: Latossolo Vermelho acriférrico; LAd: Latossolo Amarelo distrófico; Biossólido 1: biossólido equilibrado com LVwf; Biossólido 2: biossólido equilibrado com LAd; Troc.: trocável; Superf. Ox.: superfície de óxidos; MO: matéria orgânica; Ox. Mn: óxidos de Mn; Ox. Fe: óxidos de Fe malcristalizados; Fe Crist.: óxidos de Fe cristalinos; Res.: residual. 
encontrado na fração Superf. Ox. e 23 \% na Ox. Mn. Morera et al. (2001) encontraram distribuição de $\mathrm{Cu}$ semelhante à encontrada no LAd, ou seja, cerca de $56 \%$ do $\mathrm{Cu}$ estava retido na fração Superf. Ox. A maior importância da fração residual na retenção de $\mathrm{Cu}$ no LVwf deveu-se ao maior teor de argila (Quadro 1), a qual é predominantemente caulinítica (resultados não apresentados).

A adição dos condicionadores não alterou a distribuição de $\mathrm{Cu}$ no LVwf, o que ocorreu, provavelmente, porque o $\mathrm{Cu}$ encontrava-se em formas pouco móveis nas amostras-controle. Nessa situação, o efeito dos condicionadores foi menos acentuado. Em contrapartida, as quantidades de $\mathrm{Cu}$ associadas à fração residual foram aumentadas no LAd após a adição dos condicionadores (ausência de $\mathrm{Cu}$ na fração residual na amostra-controle comparado com 10-17\% do $\mathrm{Cu}$ total nas amostras tratadas com condicionadores). Em geral, a disponiblidade de Cu parece pouco alterada pelas variações do meio (Smith, 1994a), tais como a adição de condicionadores, graças à elevada afinidade dos colóides em adsorvê-lo (Morera et al., 2001; Silveira et al., 2002). Nos biossólidos, o $\mathrm{Cu}$ estava retido principalmente nas frações Ox. Fe, Ox. Mn, Superf.
Ox. e MO (Figura 5c,d). Não houve alteração na distribuição de $\mathrm{Cu}$ com a adição dos condicionadores.

\section{Distribuição de cádmio na fase sólida}

Em ambos os solos, o Cd foi encontrado, sobretudo nas frações Superf. Ox. e Troc. (Figura 6a, b). No LVwf, a soma dessas frações correspondeu a cerca de $64 \%$ do Cd total e, no LAd, ao redor de $96 \%$. O uso dos condicionadores reduziu as quantidades desse metal na forma trocável e aumentou, principalmente, a fração Superf. Ox. Isso pode representar uma redução na disponiblidade do metal às plantas, uma vez que, embora a fração Superf. Ox. possa ser solubilizada, ela é menos prontamente disponível do que a fração trocável. Quando comparado à amostra-controle, o tratamento com $\mathrm{CaCO}_{3}$, por exemplo, resultou na redução do Cd-Troc. de $45 \%$ do total para zero no LVwf e de $82 \%$ do total para $11 \%$ no LAd. Embora apresentem eficiência menos marcante que o $\mathrm{CaCO}_{3}$, a $\mathrm{HA}$, o $\mathrm{CaSO}_{4}$ e o $\mathrm{KH}_{2} \mathrm{PO}_{4}$ também promoveram redução nas quantidades trocáveis de $\mathrm{Cd}$. $\mathrm{AHA}(\mathrm{pH} 6)$, por exemplo, reduziu as percentagens de Cd-Troc. em 42 e $28 \%$ no LVwf e no LAd, respectivamente, em relação à amostra-controle. Isso significa que, (a) $\mathbf{L V w f}$

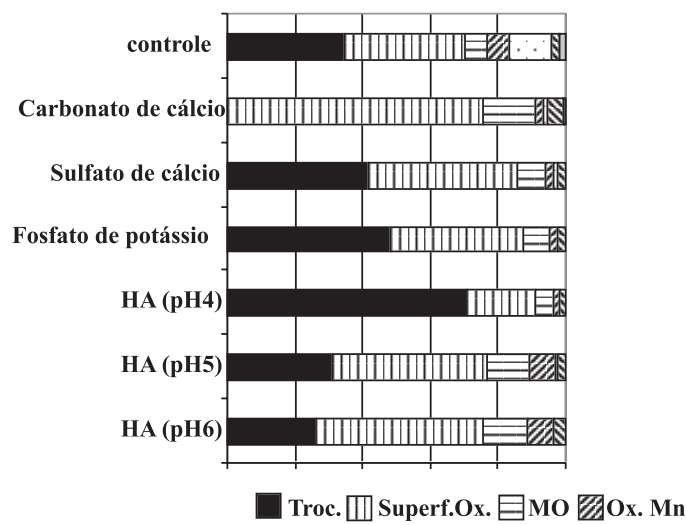

(c) Biossólido 1

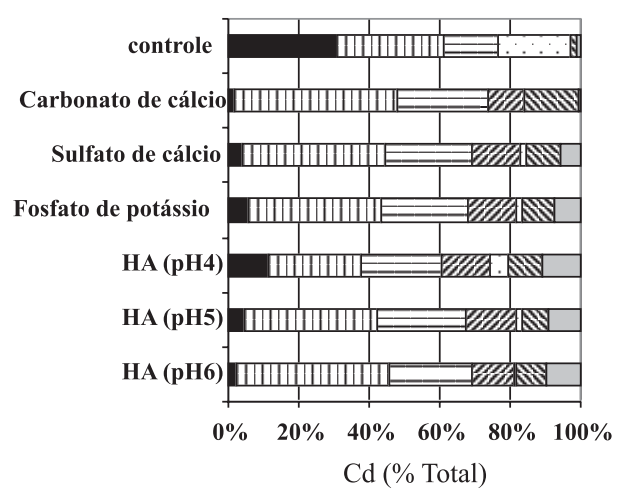

(b) LAd

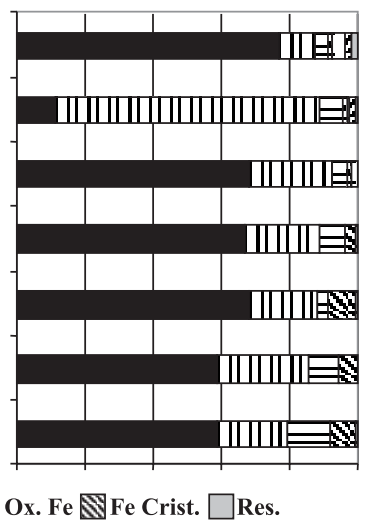

(d) Bios sólido 2

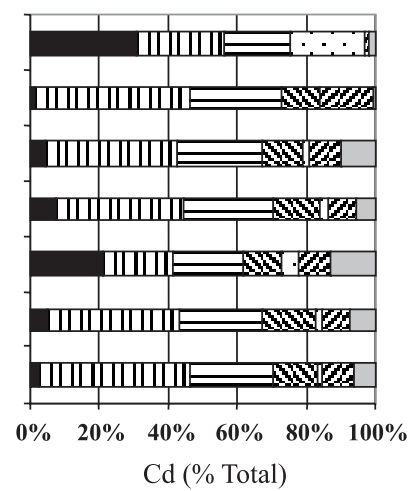

Figura 6. Distribuição das formas de cádmio nas amostras de solo e biossólido após os equilíbrios nas câmaras duplas de difusão. LVwf: Latossolo Vermelho acriférrico; LAd: Latossolo Amarelo distrófico; Biossólido 1: biossólido equilibrado com LVwf; Biossólido 2: biossólido equilibrado com LAd; Troc.: trocável; Superf. Ox.: superfície de óxidos; MO: matéria orgânica; Ox. Mn: óxidos de Mn; Ox. Fe: óxidos de Fe mal cristalizados; Fe Crist.: óxidos de Fe cristalinos; Res.: residual. 
dependendo do nível de contaminação dos solos e dos metais presentes, a escolha do condicionador pode ser realizada de acordo com o custo ou com as práticas de manejo adotadas na área, tal como a necessidade de calagem e, ou, correção com $\mathrm{CaSO}_{4}$. Assim como o observado por Xu \& Schwartz (1994), nos valores mais elevados de $\mathrm{pH}$, houve maior adsorção de $\mathrm{Cd}$. No $\mathrm{pH} 5$, não houve diferenças marcantes na distribuição de $\mathrm{Cd}$ nas amostras tratadas com $\mathrm{HA}, \mathrm{CaSO}_{4}$ ou $\mathrm{KH}_{2} \mathrm{PO}_{4}$. Portanto, a redução do $\mathrm{pH}$ para 4 no tratamento com HA resultou em maior quantidade de Cd-Troc. em relação à amostra-controle, possivelmente pelo efeito do $\mathrm{pH}$. Na amostra tratada com HA ( $\mathrm{pH} 4)$, houve maior dissolução do biossólido em relação à amostracontrole e, conseqüentemente, maior quantidade de $\mathrm{Cd}$ foi disponibilizada do biossólido para solução. Nessas condições, o $\mathrm{Cd}$ foi retido nos solos, principalmente na forma trocável.

Apesar de os valores absolutos de $\mathrm{Cd}$ nos biossólidos não terem sido alterados com a adição dos condicionadores, sua distribuição foi afetada sensivelmente (Figura 6c,d). Assim como observado por Fang \& Wong (1999), o tratamento com $\mathrm{CaCO}_{3}$ promoveu redução na percentagem de $\mathrm{Cd}$ retido na fração trocável. Cerca de 10-12\% do Cd total na amostra-controle estava presente na fração Troc., enquanto esse valor diminuiu para $2 \%$ na amostra tratada com $\mathrm{CaCO}_{3}$. A quantidade de $\mathrm{Cd}$ na fração Troc. foi superior à da amostra-controle apenas na amostra equilibrada com o LVwf que recebeu o tratamento HA ( $\mathrm{pH} 4)$, sendo $12 \%$ do Cd total na amostra-controle e $21 \%$ na amostra tratada com HA (pH 4).

\section{CONCLUSÕES}

1. Os efeitos da adição de condicionadores de solo na remoção de $\mathrm{Zn}, \mathrm{Cu}$, e $\mathrm{Cd}$ da solução foram dependentes do metal e do tipo de solo.

2. Com exceção do tratamento que recebeu a HA ( $\mathrm{pH}$ 4), os condicionadores de solo foram eficientes na remoção de Zn e de Cd solução. Em contrapartida, os efeitos da adição de condicionadores de solo na remoção de $\mathrm{Cu}$ solúvel foram pouco pronunciados graças à baixa concentração desse metal em solução. $\mathrm{O} \mathrm{CaCO}_{3}$ foi o condicionador mais eficiente na imobilização dos metais, seguido da hidroxiapatita ao $\mathrm{pH} 6$.

3. A quantidade dos metais na fração trocável foi, em geral, diminuída, enquanto a fração dos metais ligados à superfície dos óxidos/carbonatos foi aumentada após adição dos condicionadores no solo, com exceção do tratamento que recebeu a $\mathrm{HA}(\mathrm{pH} 4)$.

4. Para o LVwf, a adição de $\mathrm{CaSO}_{4}$ e de $\mathrm{KH}_{2} \mathrm{PO}_{4}$ não reduziu as concentrações de $\mathrm{Zn}$ e $\mathrm{Cd}$ associadas à fração trocável desse solo. Entretanto, esses condicionadores foram eficientes na redução de Zn e Cd trocável no LAd.
5. O Cu foi retido em formas poucos móveis no solo e no biossólido (residual, óxidos de Fe e Mn), enquanto $\mathrm{Zn}$ e Cd encontraram-se em formas mais prontamente disponíveis (trocável e associado à superfície dos óxidos) em ambos os Latossolos.

\section{LITERATURA CITADA}

BOISSON, J.; RUTTENS, A.; MENCH, M. \& VANGRONSVELD, J. Evaluation of hydroxiapatite as a metal immobilizing soil additive for the remediation of polluted soils. Part 1. Influence of hydroxyapatite on metal exchangeability in soil, plant growth and plant metal accumulation. Environ. Poll., 104: 225-233, 1999.

BROWN, S.; CHRISTENSEN, B.; LOMBI, E.; MCLAUGHLIN, M.; MCGRATH, S.; COLPAERT, J. \& VANGRONVELD, J. An inter-laboratory study to test the ability of amendments to reduce the availability of $\mathrm{Cd}, \mathrm{Pb}$, and $\mathrm{Zn}$ in situ. Envir. Poll., 138: 34-45, 2005.

CAMARGO, O.A.; MONIZ, A.C.; JORGE, J.A. \& VALADARES, J.M.A.S. Métodos de análise química, mineralógica e física de solos do IAC. Campinas, Instituto Agronômico de Campinas, 1986. 94p. (Boletim Tecnico, 106)

CANDELARIA, L.M. \& CHANG, A.C. Cadmium activities, solution speciation, and solid phase distribution of $\mathrm{Cd}$ in cadmium nitrate and sewage sludge-treated soil systems. Soil Sci., 162:722-732, 1997.

CERRI, C.E.P.; SPAROVEK, G.; BERNOUX, M.; EASTERLING, W.E.; MELILLO, J.M. \& CERRI, C.C. Tropical agriculture and global warming: Impacts and mitigation options. Sci. Agric., 64:83-99, 2007.

CORNU, S.; NEAL, C.; AMBROSI, J.P.; WHITE HEAD, P.; NEAL, M.; SIGOLO, J. \& VACHIER, P. The environment impact of heavy metals from sewage sludge in Ferrasols (São Paulo, Brazil). Sci. Total Environ., 271:27-48, 2001.

DePINTO, J.V. An experimental apparatus for evaluating kinetics of available phosphorus release from aquatic particulates. Water Res., 16:1065-1070, 1982.

FANG, M. \& WONG, J.W.C. Effects of lime amendment on availability of heavy metals and maturation in sewage sludge composting. Environ. Poll., 106:83-89, 1999.

FONTES, M.P.F. \& ALLEONI, L.R.F. Electrochemical attributes and availability of nutrients, toxic elements, and heavy metals in tropical soils. Sci. Agric., 63:589-608, 2006.

GRIFFIN, R.A. \& JURINAK, J.J. Estimation of ion activity coefficients from electrical conductivity of natural aquatic systems and soil extracts. Soil Sci., 116:26-30, 1973.

GUSTAFSSON, J.P. Visual MINTEQ, ver 2.32. Stockholm, Royal Institute of Technology, Department of Land and Water Resources Engineering, 2005. Disponível em: $<$ http://hem.bredband.net/b108693>. 
HAMON, R.E.; McLAUGHLin, M.J. \& COZENS, G. Mechanisms of attenuation of metal availability in situ remediation treatments. Environ. Sci. Technol., 36:3991 3996, 2002.

KABATA-PENDIAS, A. \& PENDIAS, H. Trace elements in soils and plants. Boca Raton, CRC Press, 1987. 315p.

KANABO, I.A.K. \& GILKES, R.J. The role of soil $\mathrm{pH}$ in the dissolution of phosphate rock fertilizers. Fert. Res., 12:165$173,1987$.

KELLER, A.; STEIGER, B.V.; van der ZEE, S.E.A.T. \& SCHULIN, R. A stochastic empirical model for regional heavy-metal balances in agroecosystems. J. Environ. Qual., 30:1976-1989, 2001.

MA, Q.Y.; TRAINA, S.J. \& LOGAN, T.J. In situ lead immobilization by apatite. Environ. Sci. Technol., 27:18031810, 1993.

MA, Q.Y.; TRAINA, S.J.; LOGAN, T.J. \& RYAN, J.A. Effects of aqueous $\mathrm{Al}, \mathrm{Cd}, \mathrm{Cu}, \mathrm{Fe}(\mathrm{II}), \mathrm{Ni}$ and $\mathrm{Zn}$ on $\mathrm{Pb}$ immobilization by hydroxiapatite. Environ. Sci. Technol., 28:1219-1228, 1994.

MORERA, M.T.; ECHEVERRÍA, J.C.; MAZKIARÁN, C. \& GARRIDO, J.J. Isotherms and sequential extraction for evaluating sorption and distribution of heavy metals in soils. Environ. Poll., 113:135-144, 2001.

RHOADES, J.D. Salinity: Electrical conductivity and total dissolved solids. In: SPARKS, D.L., eds. Methods of soil analysis - part 3, chemical methods. Madison, Soil Science Society of America, 1996. p.417-435.

ROSS, S.M. Retention, transformation and mobility of toxic metals in soil-plant systems. In: ROSS, S.M., ed. Toxic metals in soil-plant system. Chichester, John Wiley, 1994. p.63-152.

SALAM, A.K. \& HELMKE, P.A. The $\mathrm{pH}$ dependence of free ionic activities and total dissolved concentrations of copper and cadmium in soil solution. Geoderma, 83:281-291, 1998.
SHUMAN, L.M. Effect of phosphorus level on extractable micronutrients and their distribution among soil fractions. Soil Sci. Soc. Am. J., 52:136-141, 1988.

SILVEIRA, M.L.; ALLEONI, L.R.F.; O'CONNOR, G.A. \& CHANG, A.C. Heavy metal sequential extraction methods - A modification for tropical soils. Chemosphere, 64:19291938, 2007b.

SILVEIRA, M.L.; CHANG, A.C.; ALLEONI, L.F.R.; O'CONNOR, G.A. \& BERTON, R. Metal-associated forms and speciation in biosolids-amended Oxisols. Comm. Soil Sci. Plant Anal., 38:851-869, 2007a.

SILVEIRA, M.L.A.; ALLEONI, L.R.F. \& GUILHERME, L.R.G. Biosolids and heavy metals in soils. Sci. Agric., 60:793806, 2003.

SILVEIRA, M.L.A.; ALLEONI, L.R.F.; CASAGRANDE, J.C. \& CAMARGO, O.A. Copper adsorption in oxidic soils after removal of organic matter and iron oxides. Comm. Soil Sci. Plant Anal., 33:3581-3592, 2002.

SMITH, S.T. Effect of soil pH on availability to crops of metals in sewage sludge-treated soils. I. Nickel, copper and zinc uptake and toxicity to ryegrass. Environ. Poll., 85:321327, 1994a.

SMITH, S.T. Effect of soil pH on availability to crops of metals in sewage sludge-treated soils. II. Cadmium uptake by crops and implications for human dietary intake. Environ. Poll., 86:5-13, 1994b.

USEPA. U.S. Environmental Protection Agency. Method 3052: Microwave assisted acid digestion of siliceous and organically based matrices (compact disc). Washington, 1996.

WONG, J.W. \& FANG, M. Effects of lime addition on sewage sludge composting process. Water Res., 34:3691-3698, 2000 .

XU, Y. \& SCHWARTZ, F.W. Sorption of $\mathrm{Zn}^{2+}$ and $\mathrm{Cd}^{2+}$ on hydroxyapatite surfaces. Environ. Sci. Technol., 28:14721480, 1994. 